\title{
Zeitschrift für Erziehungswissenschaft 2011 - Ein Jahresrückblick
}

\author{
Harm Kuper
}

Liebe Leserinnen und Leser der Zeitschrift für Erziehungswissenschaft,

zum Start in den 15. Jahrgang der ZfE möchte ich den vorangegangenen 14. Jahrgang noch einmal Revue passieren lassen. Der 14. Jahrgang ist der erste der ZfE, der mit einem Jahresrückblick eingeleitet wurde. Er sollte Ihnen Gelegenheit geben, das Profil eines Jahres der Zeitschrift auf wenigen Seiten kondensiert in Augenschein zu nehmen und mit Ihrer Sicht auf die aktuellen Entwicklungen der Erziehungswissenschaft und die daraus sich ergebenden Erwartungen an ein erziehungswissenschaftliches Fachjournal abzugleichen.

Zudem entspricht es dem Wunsch der Herausgeberinnen und Herausgeber der ZfE, wenn Sie sich von der Lektüre eines Jahresrückblicks dazu motivieren lassen, selbst einen Artikel einzureichen: sei es, um mit einem Beitrag aus Ihrer Forschung eine Facette zu einem Schwerpunkt eines vorangegangenen Hefts beizusteuern oder um einem bislang vernachlässigten Thema Geltung zu verschaffen. Zwar muss ich Ihnen einen wissenschaftlich empirischen Beleg schuldig bleiben, aber ich habe den sicheren Eindruck, der Jahresrückblick auf das Jahr 2010 hat in diesem Sinne zu einer Bereicherung des Spektrums geführt, das von den 2011 eingereichten Manuskripten abgedeckt wurde. Verstehen Sie den Jahresrückblick daher gerne als eine Einladung, die in der ZfE geführte Diskussion um die theoretische Ordnung der Gegenstände sowie die Befunde und methodischen Ansprüche der Forschung in der Erziehungswissenschaft und ihren Nachbarwissenschaften mitzugestalten.

Die Schwerpunkte der ZfE des Jahres 2011 - Start in den Lehrerberuf, Emotionen, Bildungsungleichheit in Schule und Ausbildung, Aspekte der Jugendforschung - reflektieren einige der Themen, die in den Diskussionen um das disziplinäre Selbstverständnis der Erziehungswissenschaft, ihrer Standortbestimmung im Rahmen interdisziplinärer Bildungsforschung und der Rezeption ihrer Forschungsbefunde auch über die Fachwissenschaften hinaus Bedeutung tragen.

Online publiziert: 02.02 .2012

(C) VS Verlag für Sozialwissenschaften 2012

Prof. Dr. H. Kuper $(\triangle)$

Arbeitsbereich Weiterbildung und Bildungsmanagement, Freie Universität Berlin, Arnimallee 12, 14195 Berlin, Deutschland

E-Mail: harm.kuper@fu-berlin.de 
Der Schwerpunkt Start in den Lehrerberuf greift ein hochaktuelles Forschungsthema auf, mit dessen Bearbeitung die Erziehungswissenschaft via Reflexion Verantwortung für die Entwicklung im Bildungssystem übernimmt. Die Lehrerbildung und der Übertritt in den Lehrerberuf sind aus bildungspolitischer und professioneller Perspektive als organisatorische Schaltstellen für Reformen im Schulsystem identifiziert; die Erziehungswissenschaft befindet sich über ihren Beitrag in der universitären Lehrerbildung und über ihre diesbezügliche Forschungsleistung sowohl in einer praktischen als auch in einer reflexiven Einstellung zum Übergang in den Lehrerberuf. Der Überblicksbeitrag von Tynjälä und Heikkinen (2011) verdeutlicht diese im System selbst erbrachte Forschungsund Reflexionsleistung der Erziehungswissenschaft; die Theoretisierung der Praxis, in die junge Lehrerinnen und Lehrer starten, und die Entschlüsselung ihrer ,,practice architectures“ werden gleichermaßen zur Grundlage für die Identifikation von Forschungsfragen und von Kriterien der Beurteilung einer gelingenden Professionalisierung in der Startphase des Lehrerberufs. Die anschließenden Beiträge nehmen spezifische Aspekte auf; sie berichten von Studien, in denen organisatorische Bedingungen des Berufseinstiegs und Entwicklungen in der frühen Phase der Professionalisierung untersucht werden. Richter et al. (2011) gehen im Rahmen der Studie COACTIV den Effekten sozialer Unterstützung beim Einstieg in das Lehramt durch Mentorinnen und Mentoren nach; Kreis und Staub (2011) zeigen in ihrem Beitrag die Verzahnung praktischer Innovation mit einem quasi-experimentellen Forschungsdesign anhand einer Studie über fachspezifisches Unterrichtscoaching auf; der Beitrag von Baer et al. (2011) stellt Ergebnisse einer Längsschnittuntersuchung zur Entwicklung von Unterrichtskompetenzen in der Phase des Berufseinstiegs dar. Die drei Beiträge setzen in der Lehrer-(bildungs-)forschung Maßstäbe hinsichtlich der Datenbasis, der Forschungsdesigns und der theoretischen Verortung in aktuellen Paradigmen.

Der Schwerpunkt des Hefts 2-2011 schließt für die Erziehungswissenschaft ein Thema auf, das insbesondere in der Psychologie, aber auch in den Neurowissenschaften und den Kulturwissenschaften in erheblichem Maße zur Bündelung von Forschungsaktivitäten geführt hat: Emotionen. Ein zentrales Erkenntnisinteresse der Forschung über Emotionen zielt darauf, Kenntnisse über die kulturellen Codierungen der affektiven Grundlagen von Kommunikation und sozialem Handeln zu erlangen. Hier setzt die Rezeption der Emotionsforschung in den Erziehungswissenschaften an. Eine grundlagentheoretisch aufgearbeitete Annäherung bietet der Beitrag von von Scheve (2011). Er sieht Emotionen in einer zweifachen Relation zu sozialen Entitäten - sie werden in sozialen Umwelten konstituiert und erfüllen soziale Funktionen. Von Scheve zeigt diese Zusammenhänge in Hinblick auf Mechanismen der Inklusion und Exklusion auf. Damit besteht ein unmittelbarer Anschluss an erziehungswissenschaftliche Fragen nach Bedingungen der Bildungsbeteiligung. Zirfas (2011) spürt begriffs- und ideengeschichtliche Wurzeln des pädagogischen Nachdenkens über Glück auf. Indem er die u. a. mit moralischen und utopischen Konnotationen aufgeladene Reflexion auf die Emotion Freude bis in gegenwärtige Diskurse über Spaß und Wohlbefinden verfolgt, zeigt er das Potenzial des Leitbegriffs Emotion für die Analyse normativer Vorstellungen über Bildung und Erziehung auf. Wertenbruch und Röttger-Rössler (2011) nehmen den Impuls von von Scheve in einer empirischen Fallstudie auf. Sie untersuchen das Erleben von Scham im Schulalltag unter den Aspekten der sozialen Funktion und der Differenz kultureller Orientierungen. Über die Adaption des 
Themas Emotion für die erziehungswissenschaftliche Forschung hinaus erinnert dieser „emotionsethnologische“ Beitrag an die ethnographische Tradition der Schulforschung. Im Rahmen der ZfE-Edition ist der Schwerpunkt ein appetizer für das im Jahr $2012 \mathrm{zu}$ erwartende Sonderheft „Die Bildung der Gefühle“.

Mit dem Heft 3-2011 startet ein neues Beitragsformat der ZfE unter dem Rubriktitel Standpunkt. Dieses Format bildet zukünftig ein Forum für Beiträge, die anlässlich besonderer Ereignisse und Entwicklungen in der Erziehungswissenschaft, gleichsam mit Distanz zu den Projekten und Produkten der normal science entstehen. Beiträge für die Rubrik Standpunkt sollen nach dem Verständnis der Herausgeberinnen und Herausgeber von besonderer Relevanz für das disziplinäre Selbstverständnis der Erziehungswissenschaft, für disziplin- und bildungspolitische Diskussionen oder paradigmatische Entwicklungen in der erziehungswissenschaftlichen Forschung sein. In besonderer Weise entspricht diesem Verständnis der Beitrag von Tenorth (2011), der das „Thema“ Bildung historisch aufklärt und mit den „Erwartungen an eine Theorie des Aufwachsens in Gesellschaften“ (a. a. O., S. 359) konfrontiert. Positionsbestimmungen in grundlegenden Fragen erzeugen oft Widerspruch - auch in diesem Fall folgt eine Auseinandersetzung, die wir in einem der kommenden Hefte dokumentieren.

Im Lichte des Standpunktes wird die Tragweite der weiteren Beiträge des Heftes 32011, die Befunde der normal science unter dem Schwerpunkt „Bildungsungleichheit in Schule und Ausbildung“ präsentieren, in besonderer Weise deutlich. Sie spiegeln nicht nur die enorme Produktivität empirischer Arbeiten zur Bildungsungleichheit und die reichhaltigen Möglichkeiten der in den letzten Jahren entwickelten Datengrundlagen für die Bildungsforschung; darüber hinaus tragen die Beiträge grundsätzlich zur Aufklärung kausaler Effekte auf die Bildungsbeteiligung und den Bildungserfolg bei, von denen nicht mehr abstrahiert werden kann, sobald Bildung sozial kontextuiert betrachtet wird. So liefert Schneider (2011) neue Befunde zum Einfluss des sozioökonomischen Status der Eltern und des Migrationshintergrunds auf Schullaufbahnempfehlungen; Walter (2011) zeigt relative Erfolge einer Schülerpopulation mit Migrationshintergrund am Beispiel der vietnamesischen und der philippinischen Jugendlichen auf; die Studie von Buch et al. (2011) deckt Gedächtnisstrukturen im Verlaufe von Bildungskarrieren auf, die zur Kumulation schulischer Benachteiligung beim Übergang in die Erwerbstätigkeit führen; Trautwein et al. (2011) zeigen in ihrer Studie auf, wie über den Weg der Organisation des Schulsystems - hier die Öffnung der gymnasialen Oberstufe für Realschüler - Einflüsse der sozialen Herkunft auf die Bildungsbeteiligung vermindert werden können; Kramer et al. (2011) kennzeichnen Hochschulen unterschiedlichen Typs als „differenzielle Lernund Entwicklungsmilieus“, in denen Leistungsvoraussetzungen, Interessen und sozialer Hintergrund der Studierenden systematisch variieren.

Die Beiträge im Schwerpunkt des Heftes 4-2011 greifen Aspekte der Jugendforschung auf. Jugend - als ein in erster Linie über das Lebensalter definiertes Forschungsgebiet weist eine Vielzahl thematischer Bezüge auf und wird von diversen theoretischen Standpunkten aus erschlossen, sodass eine eindeutige Verortung im disziplinären Kontext der Erziehungswissenschaft unangemessen wäre. Pfaff (2011) reflektiert diese Situation der Jugendforschung auf der Grundlage einer umfassenden Kenntnis des Diskussions- und Forschungsstandes in einem Stichwortartikel. Sie identifiziert thematische Schwerpunkte, theoretische Grundbegriffe und Trends einer interdisziplinär verstandenen Jugendfor- 
schung. Die weiteren Beiträge schlagen thematisch Brücken in den disziplinären Zuständigkeitsbereich der Erziehungswissenschaft. Gille et al. (2011) berichten aus einer Studie zur zivilgesellschaftlichen Beteiligung im Alter von 13 bis 32 Jahren; sie klären über die Nutzung von Bildungsressourcen auf, die außerhalb der formalen Organisation des Bildungssystems liegen. King et al. (2011) leisten auf der Grundlage einer qualitativen Studie einen maßgeblichen Beitrag zur Theoretisierung des oft bestätigten Befunds der Bildungsbenachteiligung von (männlichen türkischen) Kindern aus Migrantenfamilien; sie explizieren ein Konzept der Bildungsaspiration, das Muster der intergenerationalen Transmission von Bildungschancen berücksichtigt. Lex und Zimmermann (2011) klären die Dynamik der Bewältigung einer der zentralen Herausforderungen des Jugendalters - des Übergangs in berufliche Ausbildung - auf; methodisch und systematisch besonders hervorzuheben sind dabei das Vorgehen, in dem die Gesamtdynamik aus Angaben zu einzelnen Übergangsepisoden herausgearbeitet wird.

Auch die Beiträge im „Allgemeinen Teil“ der ZfE 2011 reflektieren die forschungsmethodische Pluralität der Erziehungswissenschaft, ihre thematische Dynamik zwischen paradigmatischer Engführung und theoriegenerierendem, explorativem Charakter der Forschung sowie ihre Oszillation zwischen der Außenreferenz zu den Bezugsproblemen professioneller Arbeit im Bildungssystem und der Selbstreferenz zu inner- und interdisziplinären Diskussionen. Die Qualität und die Reichweite der ZfE werden nicht zuletzt an der Zahl der Abrufe von Beiträgen aus dem Internet über Springer-Link deutlich. Sie hat für den Jahrgang 2011 erstmal die hoch gelegte Marke von 100.000 überschritten.

Im Namen der Herausgeberinnen und Herausgeber danke ich den Autorinnen und Autoren und Ihnen - liebe Leserinnen und Lesern - herzlich für Ihre Mitwirkung am Erfolg der Zeitschrift für Erziehungswissenschaft und freue mich auf viele weitere Beiträge aus Ihrer Forschung.

Ihr

Harm Kuper

\section{Literatur}

Baer, M., Kocher, M., Wyss, C., Guldimann, T., Larcher, S., \& Dörr, G. (2011). Lehrerbildung und Praxiserfahrung im ersten Berufsjahr und ihre Wirkung auf die Unterrichtskompetenzen von Studierenden und jungen Lehrpersonen im Berufseinstieg. Zeitschrift für Erziehungswissenschaft, 14, 85-117.

Buch, T., Hell, S., \& Wydra-Somaggio, G. (2011). Stigma Hauptschulabschluss? Der Einfluss der Schulbildung auf das Arbeitslosigkeitsrisiko an der zweiten Schwelle. Zeitschrift für Erziehungswissenschaft, 14, 421-443.

Gille, M., Rijke, J. de, \& Gaiser, W. (2011). Zivilgesellschaftliche Beteiligung in der Altersspanne von 13 und 32 Jahren - Entwicklung, Bedingungsfaktoren, Kontexte, Empirische Analysen auf der Basis des DJI-Surveys AID:A. Zeitschrift für Erziehungswissenschaft, 14, 551-579.

King, V., Koller, H.-C., Zölch, J., \& Carnicer, J. (2011). Bildungserfolg und adoleszente Ablösung bei Söhnen aus türkischen Migrantenfamilien. Eine Untersuchung aus intergenerationaler Perspektive. Zeitschrift für Erziehungswissenschaft, 14, 581-601. 
Kramer, J., Nagy, G., Trautwein, U., Lüdtke, O., Jonkmann, K., Maaz, K., \& Treptow, R. (2011). Die Klasse an die Universität, die Masse an die anderen Hochschulen? Wie sich Studierende unterschiedlicher Hochschultypen unterscheiden. Zeitschrift für Erziehungswissenschaft, 14, 465-487.

Kreis, A., \& Staub, F. C. (2011). Fachspezifisches Unterrichtscoaching im Praktikum. Eine quasiexperimentelle Interventionsstudie. Zeitschrift für Erziehungswissenschaft, 14, 61-83.

Lex, T., \& Zimmermann, J. (2011). Wege in Ausbildung. Befunde aus einer schrittweisen Betrachtung des Übergangsprozesses. Zeitschrift für Erziehungswissenschaft, 14, 603-627.

Pfaff, N. (2011). Stichwort: Aktuelle Entwicklungen in der Jugendforschung. Zeitschrift für Erziehungswissenschaft, 14, 523-550.

Richter, D., Kunter, M., Lüdtke, O., Klusmann, U., \& Baumert, J. (2011). Soziale Unterstützung beim Berufseinstieg ins Lehramt. Eine empirische Untersuchung zur Bedeutung von Mentoren und Mitreferendaren. Zeitschrift für Erziehungswissenschaft, 14, 35-59.

Scheve, C. von (2011). Die soziale Konstitution und Funktion von Emotion Akteur, Gruppe, normative Ordnung. Zeitschrift für Erziehungswissenschaft, 14, 207-222.

Schneider, T. (2011). Die Bedeutung der sozialen Herkunft und des Migrationshintergrundes für Lehrerurteile am Beispiel der Grundschulempfehlung. Zeitschrift für Erziehungswissenschaft, 14, 371-396.

Tenorth, H.-E. (2011). „Bildung“ - ein Thema im Dissens der Disziplinen. Zeitschrift für Erziehungswissenschaft, 14, 351-362.

Trautwein, U., Nagy, G., \& Maaz, K. (2011). Soziale Disparitäten und die Öffnung des Sekundarschulsystems. Eine Studie zum Übergang von der Realschule in die Gymnasiale Oberstufe. Zeitschrift für Erziehungswissenschaft, 14, 445-463.

Tynjälä, P., \& Heikkinen, H. L. T. (2011). Beginning teacher's transition from pre-service education to working life. Theoretical perspectives and best practices. Zeitschrift für Erziehungswissenschaft, 14, 11-33.

Walter, O. (2011). Der Schulerfolg vietnamesischer und philippinischer Jugendlicher in Deutschland. Eine Analyse auf der Grundlage der Erweiterungsstichprobe von PISA 2003. Zeitschrift für Erziehungswissenschaft, 14, 397-419.

Wertenbruch, M., \& Röttger-Rössler, B. (2011). Emotionsethnologische Untersuchungen zu Scham und Beschämung in der Schule. Zeitschrift für Erziehungswissenschaft, 14, 241-257.

Zirfas, J. (2011). Zur Pädagogik der Glücksgefühle. Ein Beitrag zur Pursuit of Happiness. Zeitschrift für Erziehungswissenschaft, 14, 223-240. 